BMJ Open

Diabetes

Research

\& Care

\title{
Clinical and biochemical profile of 786 sequential episodes of diabetic ketoacidosis in adults with type 1 and type 2 diabetes mellitus
}

\author{
Emma Ooi (D) , ${ }^{1}$ Katrina Nash, ${ }^{2}$ Lakshmi Rengarajan, ${ }^{3}$ Eka Melson, 3,4 \\ Lucretia Thomas, ${ }^{2}$ Agnes Johnson, ${ }^{2}$ Dengyi Zhou, ${ }^{2}$ Lucy Wallett, ${ }^{3}$ Sandip Ghosh, ${ }^{3}$ \\ Parth Narendran, ${ }^{3,5}$ Punith Kempegowda ${ }^{3,4}$
}

To cite: 0 oi E, Nash $\mathrm{K}$, Rengarajan $\mathrm{L}$, et al. Clinical and biochemical profile of 786 sequential episodes of diabetic ketoacidosis in adults with type 1 and type 2 diabetes mellitus. BMJ Open Diab Res Care 2021;9:e002451. doi:10.1136/ bmjdrc-2021-002451

- Additional supplemental material is published online only. To view, please visit the journal online (http://dx.doi. org/10.1136/bmjdrc-2021002451).

EO and KN are joint first authors.

PN and PK are joint senior authors.

Received 22 June 2021 Accepted 31 October 2021

Check for updates

(c) Author(s) (or their employer(s)) 2021. Re-use permitted under CC BY-NC. No commercial re-use. See rights and permissions. Published by BMJ.

For numbered affiliations see end of article.

Correspondence to Dr Punith Kempegowda; p.kempegowda@bham.ac.uk

\section{ABSTRACT}

Introduction We explored the clinical and biochemical differences in demographics, presentation and management of diabetic ketoacidosis (DKA) in adults with type 1 and type 2 diabetes.

Research design and methods This observational study included all episodes of DKA from April 2014 to September 2020 in a UK tertiary care hospital. Data were collected on diabetes type, demographics, biochemical and clinical features at presentation, and DKA management.

Results From 786 consecutive DKA, 583 (75.9\%) type 1 diabetes and $185(24.1 \%)$ type 2 diabetes episodes were included in the final analysis. Those with type 2 diabetes were older and had more ethnic minority representation than those with type 1 diabetes. Intercurrent illness (39.8\%) and suboptimal compliance (26.8\%) were the two most common precipitating causes of DKA in both cohorts. Severity of DKA as assessed by $\mathrm{pH}$, glucose and lactate at presentation was similar in both groups. Total insulin requirements and total DKA duration were the same (type 1 diabetes 13.9 units (9.1-21.9); type 2 diabetes 13.9 units (7.7-21.1); $p=0.4638)$. However, people with type 2 diabetes had significantly longer hospital stay (type 1 diabetes: 3.0 days (1.7-6.1); type 2 diabetes: 11.0 days $(5.0-23.1) ; p<0.0001)$.

Conclusions In this population, a quarter of DKA episodes occurred in people with type 2 diabetes. DKA in type 2 diabetes presents at an older age and with greater representation from ethnic minorities. However, severity of presentation and DKA duration are similar in both type 1 and type 2 diabetes, suggesting that the same clinical management protocol is equally effective. People with type 2 diabetes have longer hospital admission.

\section{INTRODUCTION}

Diabetic ketoacidosis (DKA) is a lifethreatening complication of diabetes which requires rapid assessment and treatment. ${ }^{1}$ It is characterized by the triad of hyperglycemia, acidemia and ketosis and results from insulin deficiency. ${ }^{2}$ Treatment involves correction of hyperglycemia and hypovolemia and replacement of electrolytes. ${ }^{2}$ The incidence of

\section{Significance of this study}

What is already known about this subject?

- Diabetic ketoacidosis (DKA) is generally associated with type 1 diabetes mellitus (T1DM) but can also develop in people with type 2 diabetes mellitus (T2DM).

- Common precipitants of DKA in T1DM and T2DM are intercurrent illness and suboptimal treatment.

- DKA in people with T1DM and T2DM are currently managed using the same clinical protocols.

What are the new findings?

- DKA in those with T2DM is more common in people of ethnic minority background.

- Severity of DKA at presentation as assessed by $\mathrm{pH}$, glucose and lactate does not differ between T1DM and T2DM, although people with T2DM have longer hospital stays than those with T1DM.

- Management of DKA as assessed by insulin requirements did not differ between T1DM and T2DM, but people with T1DM have more episodes of hypoglycemia during their DKA treatment.

How might these results change the focus of research or clinical practice?

- The existing DKA guidelines are appropriate for both type 1 and type 2 diabetes, thus minimizing the need to create further individualized pathways.

- Ethnic minority populations with T2DM are at greater risk of DKA and may benefit from specific education around DKA as a potential complication of their diabetes.

DKA ranges from 4.6 to 8 episodes per 1000 people with type 1 or type 2 diabetes. ${ }^{34}$ While mortality associated with DKA appears to have significantly decreased over the last 20 years (from $7.96 \%$ to $0.67 \%$ ), ${ }^{5}$ DKA still represents a considerable risk in adults, adolescents and young children. ${ }^{1}$

Diabetes is usually classified into type 1 and type 2 diabetes and most people with diabetes 
have type 2 diabetes $(90 \%-95 \%) .^{6-8}$ It is traditionally accepted that DKA is characteristic of type 1 diabetes. ${ }^{6}$ In fact, it has previously been considered that DKA is indicative, or diagnostic, of type 1 diabetes. ${ }^{1}$ However, it is also recognized that those with type 2 diabetes can also develop DKA; a retrospective cohort study recently estimated that the incidence of DKA in type 2 diabetes has increased by $4.24 \%$ annually between 1992 and $2013 .^{9}$ Unfortunately, data reporting on DKA in people with type 2 diabetes are sparse and guidelines for managing DKA in people with type 2 diabetes are based on those for type 1 diabetes.

Newton and Raskin ${ }^{10}$ studied the clinical and biochemical characteristics of 176 episodes of DKA in people with type 1 diabetes compared with 20 with type 2 diabetes. They found a greater proportion of patients with type 2 diabetes experiencing DKA were Latino-American or African-American and required a longer period of treatment to achieve ketone-free urine. Balasubramanyam et $a l^{11}$ also compared 141 people with type 1 diabetes and 55 people with type 2 diabetes presenting with DKA and identified differences in ethnicities, body mass index and age of onset at presentation. These studies are small and there are currently no published studies comparing DKA in type 1 and type 2 diabetes in the UK.

This descriptive study aimed to compare the demographics and clinical course of DKA in type 1 and type 2 diabetes. We also explored the hypothesis on whether DKA treatment algorithm is as effective in people with type 2 diabetes as in those with type 1 diabetes.

\section{METHODS}

\section{Study design, setting and population}

The study was undertaken at a large tertiary care center in West Midlands, UK, which serves an ethnically diverse population of over one million people. All people admitted with DKA from April 2014 to September 2020 were included in the study. DKA was defined as blood glucose $>11 \mathrm{mmol} / \mathrm{L}, \mathrm{pH} \leq 7.3$ or bicarbonate $\leq 15$ $\mathrm{mmol} / \mathrm{L}$ and ketonemia $\geq 3 \mathrm{mmol} / \mathrm{L}$. ${ }^{1}$ The list of people admitted with DKA was initially screened based on those who meet one of the following criteria: electronic records with $\mathrm{pH}<7.3$ and blood glucose $>11 \mathrm{mmol} / \mathrm{L}$ or referral with DKA to the specialist team or discharged with a diagnosis of DKA. ${ }^{1}$ This list was further manually verified for confirmed diagnosis of DKA and only those who met the
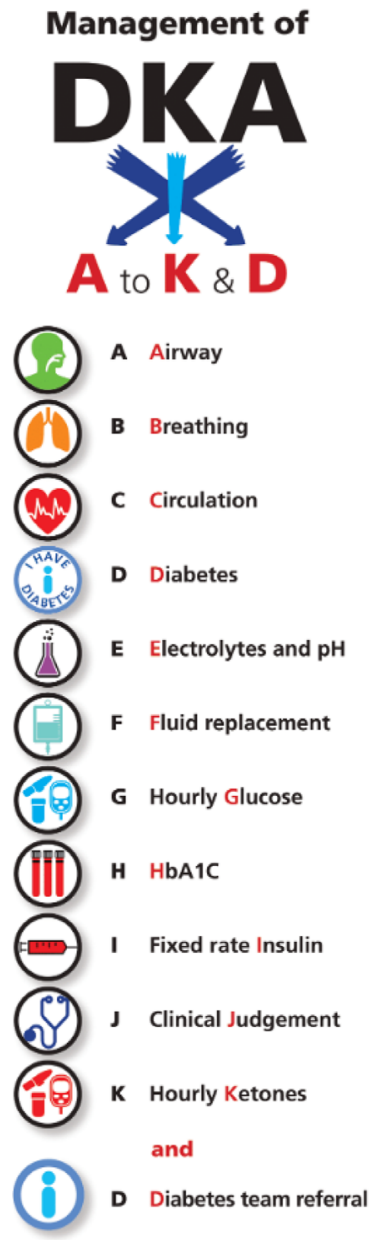

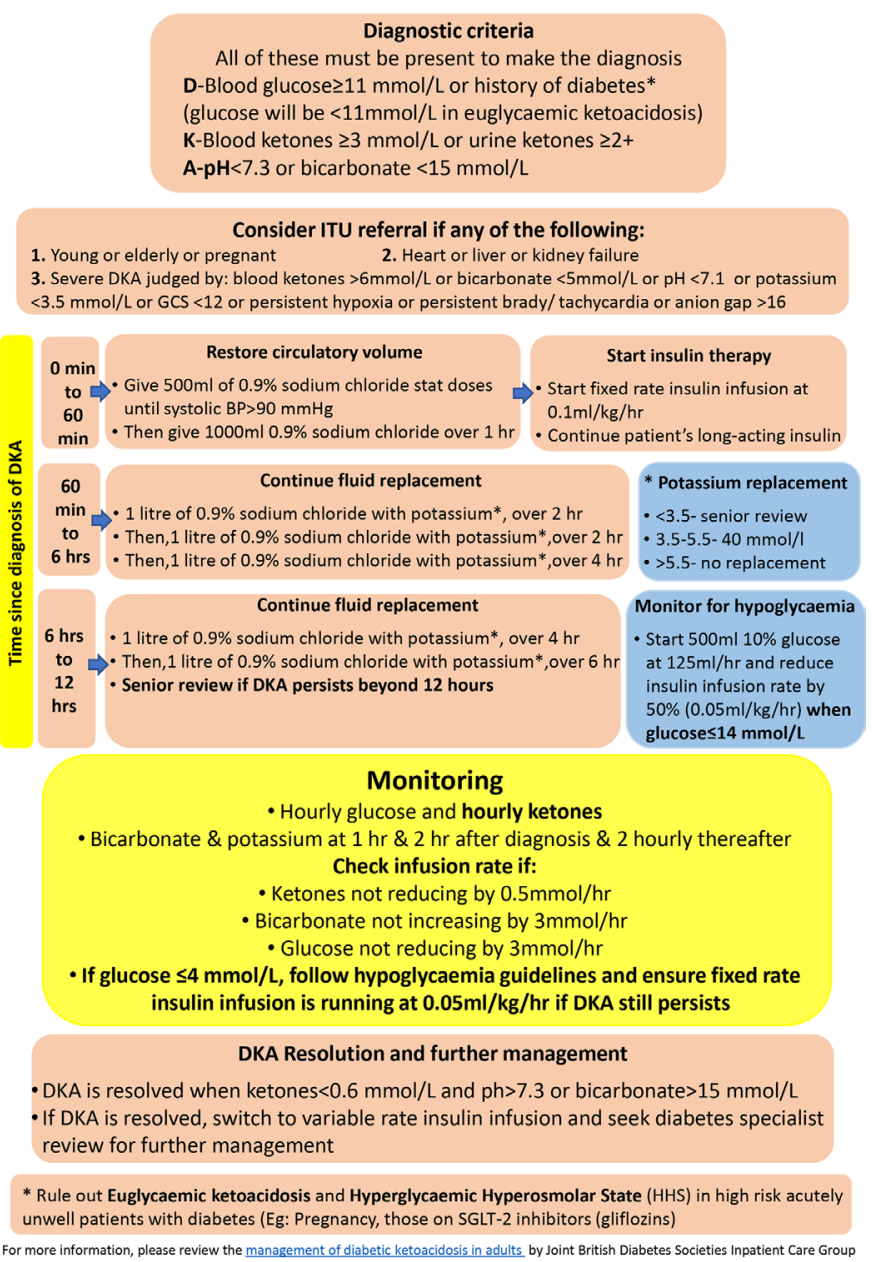

Diagnostic criteria All of these must be present to make the diagnosis D-Blood glucose $\geq 11 \mathrm{mmol} / \mathrm{L}$ or history of diabetes* will be $<11 \mathrm{mmol} /[$ in euglycaemic ketoacidosis) - $\mathrm{HH}<7.3$ or bicarbonate $<15 \mathrm{mmol} / \mathrm{L}$

Figure 1 Guidelines for DKA treatment at our center. BP, blood pressure; DKA, diabetic ketoacidosis; GCS, Glasgow Coma Scale; HbA1c, glycated haemoglobin; SGLT2, sodium-glucose cotransporter-2; ITU, intensive treatment unit. 


\begin{tabular}{|c|c|c|c|}
\hline Variable & Type 1 diabetes & Type 2 diabetes & Significance, $p$ value \\
\hline n (\%) & $583(75.9)$ & $185(24.1)$ & \\
\hline Age, median (Q1-Q3) & 28.97 (21.9-48.7) & $61.54(52.0-75.1)$ & $<0.0001^{\star}$ \\
\hline \multicolumn{4}{|l|}{ Age group, n (\%) } \\
\hline$<29$ & $289(99.7)$ & $1(0.3)$ & \\
\hline $30-49$ & $147(80.8)$ & $35(19.2)$ & \\
\hline $50-69$ & $120(59.1)$ & $83(40.9)$ & \\
\hline$>70$ & $21(24.4)$ & $65(75.6)$ & \\
\hline \multicolumn{4}{|l|}{ Sex, n (\%) } \\
\hline Male & $277(47.7)$ & $99(53.5)$ & 0.167 \\
\hline Female & $304(52.3)$ & $86(46.5)$ & \\
\hline Male:female & $1: 1.1$ & $1: 0.9$ & \\
\hline \multicolumn{4}{|l|}{ Ethnicity, n (\%) } \\
\hline White & $453(80.3)$ & $130(73.5)$ & $0.030^{*}$ \\
\hline Asian & $50(8.9)$ & $20(11.3)$ & \\
\hline Black & $32(5.7)$ & $19(10.7)$ & \\
\hline Mixed & $22(3.9)$ & $3(1.7)$ & \\
\hline Others & $7(1.2)$ & $5(2.8)$ & \\
\hline
\end{tabular}

${ }^{*} \mathrm{P}<0.05$.

Q1, first quartile; Q3, third quartile.

criteria as per national guidelines were included in the study. Classification (type 1/2) of diabetes was based on a clinical diagnosis as per National Institute of Health and Care Excellence (NICE) guidelines, although may have been confirmed through islet autoantibody or C peptide testing. Patients who had DKA were identified using an automatic monitoring system, and data regarding various aspects of DKA were collected manually. We reviewed patients' health records to confirm the type of diabetes diagnosis. Patients were excluded from analysis if the diabetes classification was unclear or if this information was unavailable.

\section{DKA management guidelines}

The guidelines for management of DKA at our center are based on the Joint British Diabetes Societies guidelines and are the same for both type 1 and type 2 diabetes. ${ }^{1}$ Treatment of DKA includes administering fluids to correct the deficit and replacement glucose infusion when serum glucose drops less than $14 \mathrm{mmol} / \mathrm{L}$ but ketosis persists. A fixed rate intravenous insulin infusion was administered based on the patient's body weight at a rate of 0.1 unit/ $\mathrm{kg} /$ hour to switch of lipolysis. Hourly glucose and ketone measurements are done to check treatment progress. Venous blood gases are done during infusion bag change to ensure the next bag of fluid has appropriate potassium replacement. While the guidelines for DKA management have evolved to ensure simplicity and end-user satisfaction over the last 7 years in our institute, the principles have not changed (figure 1).

\section{Data collection}

\section{Patient demographics}

The following variables were registered: age, sex and ethnicity. Data were based on self-identification or from medical records. Ethnicity was categorized as white, Asian, black, mixed and others.

\section{Etiology of DKA}

The precipitating cause was categorized as alcoholrelated, intercurrent illness, new diagnosis of diabetes mellitus, suboptimal compliance to diabetes treatment, cancer therapy-associated, sodium-glucose cotransporter-2 (SGLT2) inhibitors-related or unknown.

\section{DKA severity}

The following data were collected as indicators of DKA severity: serum sodium, serum potassium, serum glucose, lactate, $\mathrm{pH}$, bicarbonate, urea and serum osmolality. Serum ketone data were unavailable.

In addition, serum osmolality was calculated to explore whether there was an element of hyperosmolar hyperglycemic state in any of the episodes. HHS was defined as a calculated serum osmolality of greater than 320 $\mathrm{mOsm} / \mathrm{L}$.

\section{Progression of treatment}

Progression of DKA was measured in several ways: number of episodes of hypoglycemia (blood glucose $<4$ mmol/L), hypokalemia (potassium $<3.5 \mathrm{mmol} / \mathrm{L}$ ) and hyperkalemia (potassium $>5.5 \mathrm{mmol} / \mathrm{L}$ ) during the DKA episode. Total insulin infused was calculated as the 

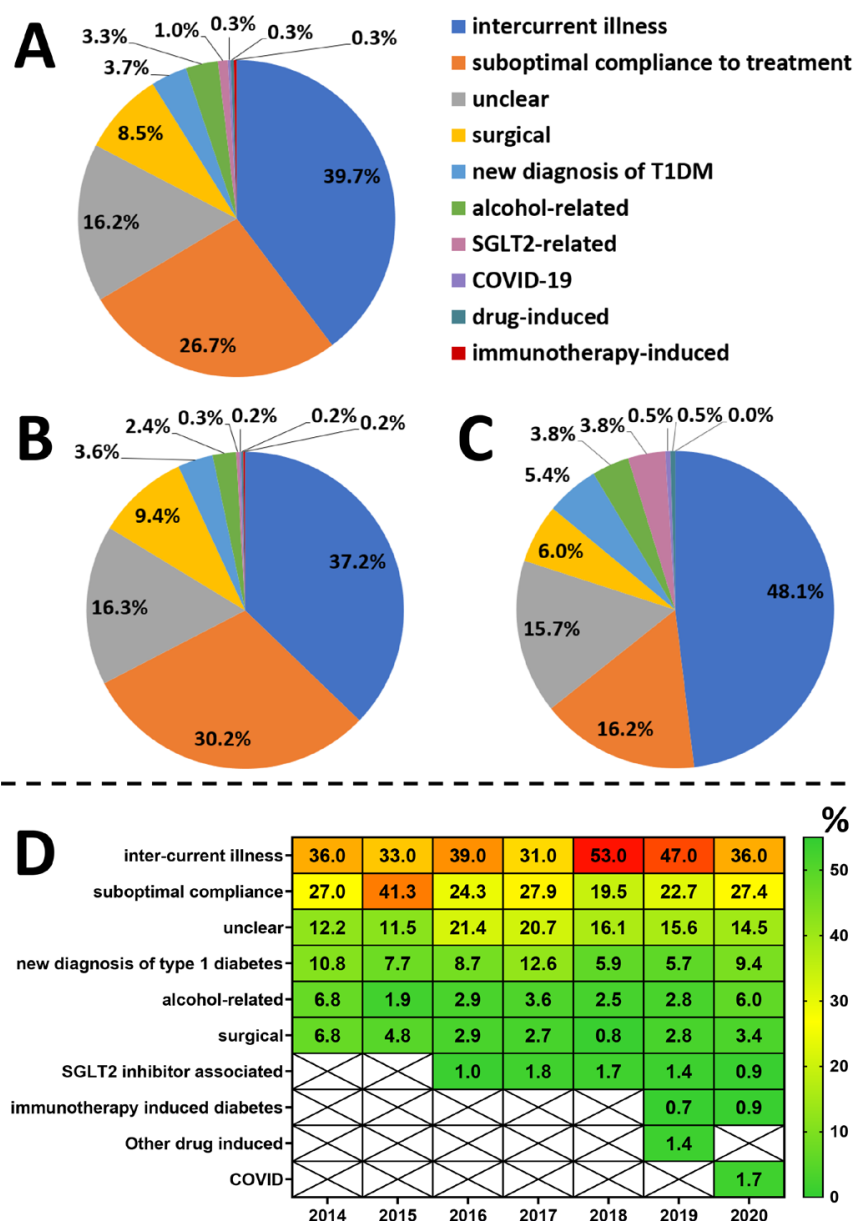

Figure 2 Proportion of various precipitating causes of DKA from 2014 to 2020: (A) overall, (B) people with type 1 diabetes, (C) people with type 2 diabetes and (D) yearwise. DKA, diabetic ketoacidosis; SGLT2, sodium-glucose cotransporter-2 inhibitors; T1DM, type 1 diabetes mellitus.

product of fixed rate intravenous insulin infusion rate and the total DKA duration. It does not include the dose of any subcutaneous basal insulin therapy that may have been initiated/maintained during the DKA episode. DKA duration (days) was calculated as the time difference between DKA diagnosis and DKA resolution $(\mathrm{pH}>7.3$ and bicarbonate $>15 \mathrm{mmol} / \mathrm{L}$; ketones $<0.6 \mathrm{mmol} / \mathrm{L})$. Length of stay (days) was calculated as the difference between admission and discharge.

\section{Data analysis}

Data were analyzed using Stata/SE V.16.1 for Mac. Descriptive statistics were used to characterize the type 1 and type 2 diabetes groups by outcome. The Shapiro-Wilk test was used to determine continuous data normality. Continuous data are presented as mean and SD if normally distributed, and as median and IQR if data were skewed. Categorical data are presented as frequency and proportions. The $\chi^{2}$ significance test, Wilcoxon rank-sum test and independent t-test were used to analyze the differences between variables, as appropriate. We used linear regression model with length of stay as outcome and type of diabetes as exposure, with age, sex and ethnicity as covariates. The findings of this model are presented as a coefficient and 95\% CI. Statistical significance was accepted at a 95\% confidence level $(\mathrm{p}<0.05)$.

Analyses on presentation, management, complications and outcome parameters were repeated in subgroups according to sex (male and female), age groups $(<30$, $30-49,50-69,>70$ ), ethnicity (white, South Asian, black, mixed, others), concurrent presence of hyperosmolality $(>320 \mathrm{mOsm} / \mathrm{L})$ and year of presentation $(2014,2015$, 2016, 2017, 2018, 2019, 2020).

\section{RESULTS}

A total of 786 DKA episodes were identified for the study. Of these, 18 were excluded due to issues with access to clinical data or lack of clarity on diabetes classification. The final analysis included 768 episodes (table 1). Shapiro-Wilk tests indicated all outcomes were nonnormally distributed and thus descriptive results are presented in median and interquartile range (IQR) and Wilcoxon rank-sum test was used.

The median age was 38.2 years (IQR 23.8-56.8) and the male to female ratio was 1:1.04. Of the patients, 583 $(78.7 \%)$ were white Caucasian, $70(9.5 \%)$ South Asian, $51(6.9 \%)$ black, $25(3.4 \%)$ mixed and $12(1.6 \%)$ other ethnicities.

Of the 768 episodes, $583(75.9 \%)$ had type 1 diabetes and $185(24.1 \%)$ had a prior diagnosis of type 2 diabetes. A summary of the baseline characteristics for the type 1 and type 2 groups is presented in table 1 . People with type 2 diabetes were older and had greater representation from ethnic minority populations compared with those with type 1 diabetes.

Intercurrent illness $(\mathrm{n}=306 / 768,39.8 \%)$, including any infection or inflammation, and suboptimal compliance to diabetes treatment $(\mathrm{n}=206 / 768,26.8 \%)$ were the two most common precipitating causes of DKA. Jointly they contributed to two-thirds of all episodes. No clear precipitating event could be identified in $16.2 \%$ ( $n=124 / 768)$ of the DKA episodes, and this was the third most common precipitant of DKA in the cohort (figure 2A). There was no difference in the common precipitating factors between the two groups (figure 2B,C). COVID-19 was identified to be the precipitating factor in two (type 1 diabetes $n=1 / 2$; type 2 diabetes $n=1 / 2)$ of the DKA episodes $(n=2 / 768$, $0.3 \%)$. Figure 2D illustrates the change in the trends of etiology over the years.

There was no significant difference in severity of DKA at presentation between type 1 and type 2 diabetes regarding $\mathrm{pH}$, glucose, lactate or osmolality. However, urea was higher in those with type 2 diabetes.

Furthermore, there was no difference in intravenous insulin requirements to treat the DKA or the total volume of fluid administered between type 1 and type 2 diabetes (table 2). DKA management was associated with more episodes of hypoglycemia in patients with type 1 diabetes than type 2 diabetes. There was no difference between 
Table 2 Biochemical profiles for severity on admission and management, complications and outcomes of DKA management by type of diabetes

\begin{tabular}{|c|c|c|c|}
\hline Parameters & Type 1 diabetes, median (Q1-Q3); n & Type 2 diabetes, median (Q1-Q3); n & $\begin{array}{l}\text { Significance, } \\
\text { p value }\end{array}$ \\
\hline $\mathrm{pH}$ & 7.22 (7.09-7.29); 270 & 7.24 (7.11-7.30); 91 & 0.3266 \\
\hline Bicarbonate (mmol/L) & 11.9 (7.1-16.8); 270 & 13.20 (7.8-17.8); 91 & 0.2192 \\
\hline Glucose (mmol/L) & $28.00(20.5-34.8) ; 274$ & 26.55 (16.2-35.1); 92 & 0.4496 \\
\hline Lactate $(\mathrm{mmol} / \mathrm{L})$ & $2.6(1.8-4.3) ; 275$ & $2.6(2.0-4.2) ; 91$ & 0.6532 \\
\hline Serum osmolality (mOsm/L) & 310.1 (300.6-320.9); 275 & 312.1 (300.1-328.8); 92 & 0.2787 \\
\hline Total insulin infused during DKA (units) & $91.6(54.5-143.9)$ & $90.2(53.6-157.4)$ & 0.5551 \\
\hline Hypoglycemic episodes (n) & $0(0-1) ; 556$ & $0(0-0) ; 173$ & $0.0056^{*}$ \\
\hline Hypokalemic episodes (n) & $0(0-1) ; 582$ & $0(0-1) ; 185$ & 0.3229 \\
\hline Hyperkalemic episodes (n) & $0(0-0) ; 582$ & $0(0-0) ; 185$ & 0.1356 \\
\hline
\end{tabular}

${ }^{*} \mathrm{P}<0.05$

DKA, diabetic ketoacidosis; Q1, first quartile; Q3, third quartile.

the groups in the number of episodes of hypokalemia or hyperkalemia during treatment for DKA.

The duration of DKA treatment was the same between patients with type 1 diabetes and type 2 diabetes, although those with type 2 diabetes required longer hospital stay (table 2). People with type 2 diabetes had stayed 4.8 (1.2-8.4) days longer compared with people with type 1 diabetes, after controlling for age, sex and ethnicity (online supplemental table 1).

\section{Sex-based differences}

Men with type 1 diabetes had higher blood glucose at presentation. Women with type 2 diabetes had higher urea and serum osmolality at presentation. There was no other difference in the biochemical parameters at presentation with DKA. Women with type 1 diabetes had more episodes of hypoglycemia during DKA treatment. There was no difference in DKA duration between men and women (online supplemental table 2).

\section{Ethnicity-based differences}

Non-Caucasian and mixed ethnicities were more likely to have type 2 diabetes (proportion of white in type 1 diabetes $80.3 \%$ vs type 2 diabetes $73.5 \%$; $\mathrm{p}=0.030$ ). In those of white Caucasian ethnicity, people with type 1 diabetes were more hyperglycemic on admission with DKA than people with type 2 diabetes; however, people with type 2 diabetes had higher urea than people with type 1 diabetes. People of Asian ethnicity with type 2 diabetes had higher lactate and urea levels on admission than people of Asian ethnicity with type 1 diabetes. Conversely, people of black ethnicity with type 2 diabetes were more hyperglycemic and had higher urea and serum osmolality on admission than people of black ethnicity with type 1 diabetes. In people of mixed ethnicity, bicarbonate was higher in those with type 2 diabetes than those with type 1 diabetes. When outcomes of DKA management were compared by type of diabetes based on ethnicity, there were no differences in total DKA duration between type 1 and type 2 diabetes, while length of hospitalization was longer in people with type 2 diabetes across all ethnicities apart from those of an Asian ethnicity (online supplemental table 3A).

Data were then compared between ethnicities by diabetes type. In those with type 1 diabetes, there were statistically significant differences in only urea levels on admission between ethnicities. However, in the type 2 diabetes group, there were statistically significant differences in bicarbonate, lactate and serum osmolality in type 2 diabetes mellitus between ethnicities. However, there were no differences in hypoglycemic, hypokalemic or hyperkalemic episodes between ethnicities in either type of diabetes. There were also no differences between ethnicities in DKA duration or length of hospitalization (online supplemental table 3B).

\section{Age-based differences}

All DKA episodes in the $<30$ years age group (online supplemental table 4) with available biochemical parameters at presentation were accounted for by people with type 1 diabetes and thus no comparison could be made between types of diabetes. There was no difference in biochemical profile on admission in the other age groups other than people with type 1 diabetes presenting more hyperglycemic and with higher serum osmolality in the 50-69 age group. People in the 50-69 age group with type 2 diabetes had more episodes of hypokalemia than people with type 1 diabetes. Otherwise, there were no differences in complications of DKA management between age groups. There was no difference in DKA duration in people with type 1 and type 2 diabetes in any of the age groups. Length of hospitalization was significantly longer in people with type 2 diabetes in the 30-49 and 50-69 age subgroups (online supplemental table $4)$. 


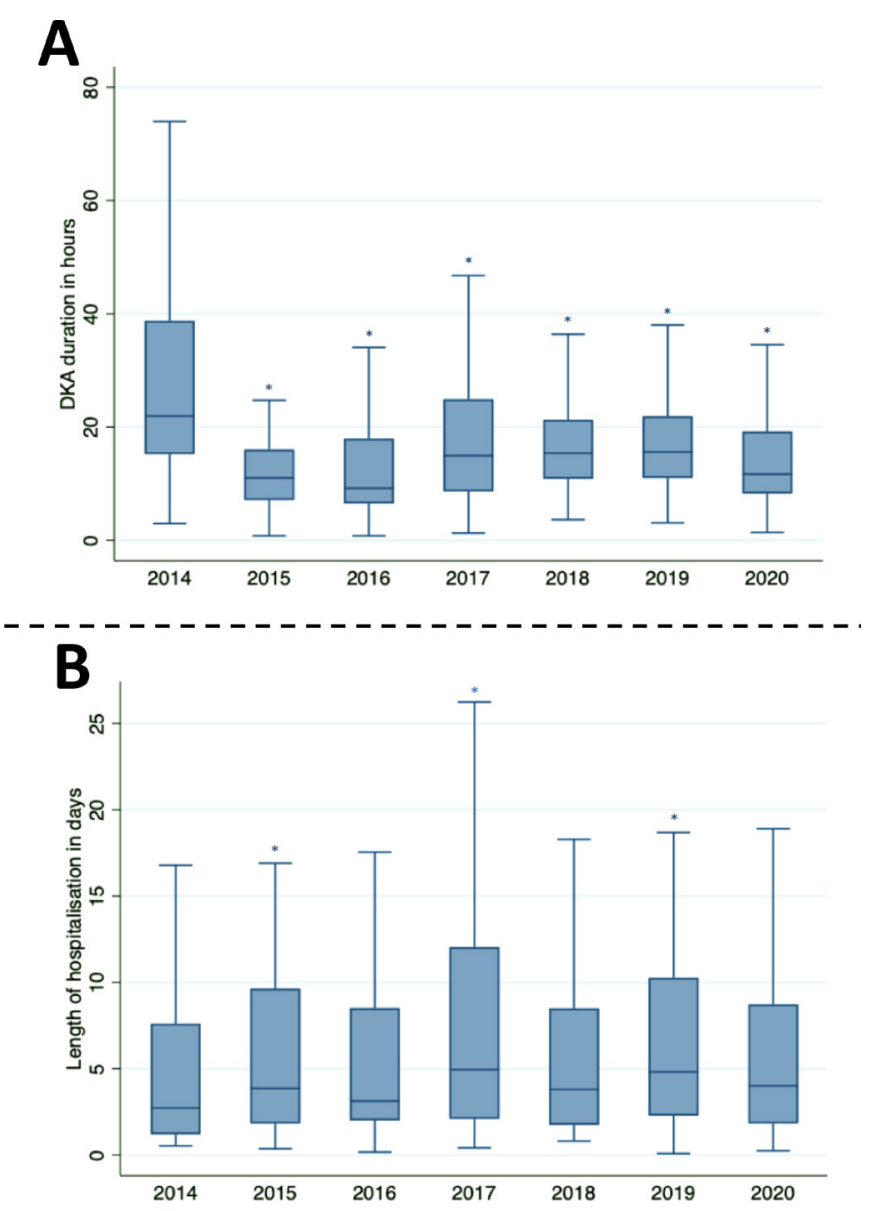

Figure 3 Differences in diabetic ketoacidosis (DKA) duration in hours (A) and length of hospitalization in days (B) based on the year of the episode compared with 2014 $\left({ }^{*} \mathrm{p}<0.05\right)$.

\section{Serum osmolality-based differences}

We also undertook an analysis of the influence of serum osmolality on the presentation and clinical outcome of DKA to explore if those who presented with a "mixed DKA/HHS' picture fared differently. ${ }^{12}$ Overall, people with a higher serum osmolality $(>320 \mathrm{mmol} / \mathrm{L})$ had higher glucose, lactate and urea levels on admission (online supplemental table 5). While there was no difference in the number of hypoglycemia episodes during the management of DKA, people with higher serum osmolality $(>320 \mathrm{mmol} / \mathrm{L})$ experienced more hyperkalemic episodes, while people with lower serum osmolality $(<320$ $\mathrm{mmol} / \mathrm{L}$ ) had more hypokalemic episodes. There was no significant difference in DKA duration between the two groups, but length of hospitalization was significantly higher in people with serum osmolality $>320 \mathrm{mmol} / \mathrm{L}$ (online supplemental table 5).

In the type 1 diabetes cohort, those with a higher serum osmolality had lower $\mathrm{pH}$, higher glucose, lactate and urea, and an increased DKA duration and length of hospital stay. People with type 2 diabetes and higher serum osmolality had higher glucose, lactate and urea, compared with those with serum osmolality $<320 \mathrm{mmol} / \mathrm{L}$. There was no difference in DKA duration and length of hospitalization between the two groups by serum osmolality (online supplemental table 5).

\section{Year-based differences}

Over the years, there was an increase in DKA admissions due to SGLT2 inhibitors and cancer immunotherapies in type 2 and type 1 diabetes, respectively, in addition to COVID-19 as a precipitating factor in 2020 (figure 2). There was a significant reduction in duration of DKA in comparison with 2014, in all subsequent years. In 2015, there was a reduction in duration of DKA by $49.8 \%$ (2014 median 21.9 (15.2-38.7); 2015 median 11.0 (7.1-16.0); $\mathrm{p}<0.0001)$. There was an upward trend from 2017 to 2019; however, DKA duration was still significantly lower in comparison with 2014. In 2020, DKA duration decreased to a median of 11.7 (IQR 9.3-19.2; $\mathrm{p}<0.0001$ ). While duration in DKA decreased over the years (figure 3A), length of hospitalization increased (figure 3B) as compared with that in 2014. This increase was significant in 2015, 2017 and 2019.

\section{DISCUSSION}

In this large observational single-center study of 786 DKA episodes, we show that DKA more commonly affects people with type 1 diabetes; however, we also noted a trend toward ethnic minorities preponderance and those of an older age, especially in type 2 diabetes. Importantly, however, nearly a quarter of admission with DKA were in people with a clinical diagnosis of type 2 diabetes. Wang $e t a l^{13}$ reported type 2 diabetes accounted a third of overall DKA cases in Swedish population. However, the results were limited by a small study population. The most common precipitants were intercurrent illness and suboptimal compliance, although no clear precipitating etiology could be ascertained for $16.5 \%$ of our cohort. There was no difference between type 1 and type 2 diabetes in DKA severity at presentation, insulin requirements for DKA treatment or complications of DKA treatment. South Asian or black ethnicities admitted with DKA were more likely to have type 2 diabetes, while those of white Caucasian ethnicity with DKA were more likely to have type 1 diabetes. People with type 2 diabetes were found to have a significantly longer hospital stay than those with type 1 diabetes. We noted a graded difference in length of hospitalization as age increased, which plateaued after 70 years.

The strengths of this study are that this is a singlecenter study of long duration with comprehensive data collection of demographics, presentation and outcome of DKA management. To our knowledge, our cohort is the largest consecutive DKA episodes ever reported, with inclusion of all people with DKA over a 6-year time period. However, we note lack of ketone measurement data, intensive care unit admission and mortality data, which limit our assessment between those with type 1 and type 2 diabetes. Previous studies showed higher mortality in type 2 diabetes with DKA compared with type 
1 diabetes ${ }^{14} 15$ and mortality is reported to relate to the precipitating cause of DKA. Additionally, antibody and C peptide data were also unavailable and thus we relied on a clinical diagnosis of type 1 diabetes and type 2 diabetes.

Our findings on the precipitating etiology are similar to previous studies which have found that intercurrent illness and suboptimal compliance were the two most common presentations. ${ }^{16}$ No identifiable precipitating factor could be found in half of patients with type 2 diabetes for DKA in a similar study in the past, akin to our study. ${ }^{11}$ Identifying the precipitating factor is important in order to avoid future episodes. We also identified a small proportion of DKA related to SGLT2 inhibitors, in line with building evidence in this drug class, ${ }^{17}{ }^{18}$ as well as increasing contribution with time from causes such as checkpoint inhibitor cancer therapy and COVID-19 virus infection. We recently reported that in people with type 1 diabetes, those with COVID-19 presented more hyperglycemic episodes, and in those with type 2 diabetes those with COVID-19 were more likely to require intensive care support and had higher mortality rates. ${ }^{19}$ Other studies have also identified changes in presenting characteristics of patients with DKA during the COVID-19 pandemic, most notably an increase in presentation of DKA in patients with type 2 diabetes. ${ }^{20} 21$

Our analysis did not show any differences in serum osmolality between DKA in type 1 and type 2 diabetes. We do show however that higher calculated osmolality across the cohort, driven by higher glucose, lactate and urea, indicated more severe dehydration and a longer length of hospitalization, but not longer DKA duration. However, those with higher serum osmolality and type 1 diabetes had an increased DKA duration and longer hospital stay when compared with those with a lower serum osmolality and type 1 diabetes.

The longer hospital stay we observed in those with type 2 diabetes may be indicative of a more complex requirement for care in this cohort. People with type 2 diabetes tend to have more comorbidities, experience macrovascular and/or microvascular complications, and have worse diabetic control compared with people with type 1 diabetes, which will add to disease burden and recovery from DKA. ${ }^{14} 22-25$ On the other hand, those with type 2 diabetes who experience DKA may represent a more complex cohort, as studies have identified increased risk of complications, such as stroke, in this group in comparison with patients with type 2 diabetes who do not experience DKA. ${ }^{26}$ Although our analyses did not find a significant difference in clinical parameters, multiple studies have indicated greater severity and mortality of DKA in patients with type 2 diabetes in comparison with those with type 1 diabetes. ${ }^{14-16}$

Balasubramanyam $e t a l^{11}$ reported a high proportion of DKA episodes in non-Caucasian adults occur in persons with type 2 diabetes, similar to our findings. ${ }^{11}$ These findings may be representative of the population diagnosed with these diseases, as minority ethnic groups are at a higher risk of type 2 diabetes. ${ }^{27}$ However, this may also be in part due to the fact that those who are South Asian or black are more likely to have worse diabetes control, have a genetic predisposition or to be of a lower socioeconomic and/or educational status, all factors which associate with an increased risk of DKA ${ }^{28-30}$ It is also increasingly recognized that non-Caucasian ethnics are more likely to be diagnosed with ketosis-prone type 2 diabetes and this may contribute to some of the DKA episodes seen. ${ }^{31}$

Over the years, our analysis identified a reduction in DKA duration but an increase in length of hospitalization. During this time period, multiple changes to DKA management have been implemented in our hospital trust as part of an ongoing quality improvement. ${ }^{32} 33$ These interventions have been effective at reducing DKA duration and have included development of a real-time audit tool, automatic referral to a specialist team, electronic surveillance of blood gas results, education and redesigning of local guidelines, and monthly feedback meetings. Nevertheless, an increase in length of hospitalization was seen which contradicts reports from other centers. ${ }^{9}$ We are still not clear of the reasons for the increased median length of stay, although this has been stable for a number of years.

The findings of this study can help inform policy and practice to reduce morbidity and mortality associated with DKA. Patient education among at-risk groups, particularly those of non-Caucasian ethnicity, can increase awareness of the potential of DKA as a complication of both type 1 and type 2 diabetes. Increasing awareness will prevent DKA occurrence and enable rapid identification of warning signs to reduce DKA-associated morbidity. Furthermore, although no differences were observed in management requirements, it is important to consider factors which may affect length of hospitalization in those with type 2 diabetes. Additionally, our subgroup analysis which confirmed that those with a higher serum osmolality are sicker may inform medical practice to alert front-line workers to patients who are likely to require more care.

\section{CONCLUSION}

Our study contributes to the understanding of DKA in people with type 1 and type 2 diabetes. We are able to confirm previous reports that DKA can present in people with a clinical diagnosis of type 2 diabetes, that DKA in type 2 diabetes is over-represented by older people and those from ethnic minority populations, and that the most common precipitants of DKA remain poor compliance with insulin and intercurrent illness.

We contribute new knowledge in that the clinical protocol for managing DKA in those with type 1 and type 2 diabetes appears equally effective, that those with higher serum osmolality appear to be more unwell and may require more support, and that new therapies such as SGLT2 inhibitors and cancer immunotherapies are increasingly contributing to the etiology of DKA in type 2 diabetes and type 1 diabetes, respectively. 
Author affiliations

${ }^{1}$ Medical School, RCSI \& UCD Malaysia Campus, Georgetown, Malaysia ${ }^{2}$ Medical School, College of Medical and Dental Sciences, University of Birmingham, Birmingham, UK

${ }^{3}$ Diabetes and Endocrinology, University Hospitals Birmingham NHS Foundation Trust, Birmingham, UK

${ }^{4}$ Institute of Metabolism and Systems Research, University of Birmingham, Birmingham, UK

${ }^{5}$ Institute of Immunology and Immunotherapy, University of Birmingham, Birmingham, UK

Acknowledgements We thank all the staff of the department of diabetes and endocrinology, University Hospitals Birmingham NHS Foundation Trust for their assistance and support to this study.

Contributors PK and PN conceptualized the study. EO, LR, EM, LT, AJ, DZ, LW and PK collected the data. EO and KN conducted statistical analysis. SG, PN and PK supervised the study. EO, KN, LR, EM and PK wrote the original draft. All authors reviewed and edited the final draft. PN and PK are the guarantors and joint senior authors taking responsibility for the content of this article.

Funding The authors have not declared a specific grant for this research from any funding agency in the public, commercial or not-for-profit sectors.

Competing interests None declared.

Patient consent for publication Not required.

Ethics approval The study was approved as part of service improvement by the Department of Information Governance, University Hospitals Birmingham NHS Foundation Trust (CARMS approval number: 12074).

Provenance and peer review Not commissioned; externally peer reviewed.

Data availability statement Data are available upon reasonable request.

Supplemental material This content has been supplied by the author(s). It has not been vetted by BMJ Publishing Group Limited (BMJ) and may not have been peer-reviewed. Any opinions or recommendations discussed are solely those of the author(s) and are not endorsed by BMJ. BMJ disclaims all liability and responsibility arising from any reliance placed on the content. Where the content includes any translated material, BMJ does not warrant the accuracy and reliability of the translations (including but not limited to local regulations, clinical guidelines, terminology, drug names and drug dosages), and is not responsible for any error and/or omissions arising from translation and adaptation or otherwise.

Open access This is an open access article distributed in accordance with the Creative Commons Attribution Non Commercial (CC BY-NC 4.0) license, which permits others to distribute, remix, adapt, build upon this work non-commercially, and license their derivative works on different terms, provided the original work is properly cited, appropriate credit is given, any changes made indicated, and the use is non-commercial. See: http://creativecommons.org/licenses/by-nc/4.0/.

ORCID iD

Emma Ooi http://orcid.org/0000-0002-6921-4395

\section{REFERENCES}

1 The management of diabetic ketoacidosis in adults. Available: http:// www.diabetologists-abcd.org.uk/JBDS/JBDS.htm [Accessed 2 Feb 2021].

2 Umpierrez GE, Kitabchi AE. Diabetic ketoacidosis: risk factors and management strategies. Treat Endocrinol 2003;2:95-108.

3 Faich GA, Fishbein HA, Ellis SE. The epidemiology of diabetic acidosis: a population-based study. Am J Epidemiol 1983;117:551-8

4 Johnson DD, Palumbo PJ, Chu CP. Diabetic ketoacidosis in a community-based population. Mayo Clin Proc 1980;55:83-8.

5 Lin S-F, Lin J-D, Huang Y-Y. Diabetic ketoacidosis: comparisons of patient characteristics. Clinical Presentations and Outcomes Today and 20 Years Ago 2005.

6 American Diabetes Association. Diagnosis and classification of diabetes mellitus. Diabetes Care 2010;33 Suppl 1:S62.

7 Chi GC, Li X, Tartof SY, et al. Validity of ICD-10-CM codes for determination of diabetes type for persons with youth-onset type 1 and type 2 diabetes. BMJ Open Diabetes Res Care 2019;7:e000547.
8 World Health Organization(WHO). The ICD-10 classification of mental and behavioural disorders. Genève: World Health Organization, 1993.

9 Zhong VW, Juhaeri J, Mayer-Davis EJ. Trends in hospital admission for diabetic ketoacidosis in adults with type 1 and type 2 diabetes in England, 1998-2013: a retrospective cohort study. Diabetes Care 2018;41:1870-7.

10 Newton CA, Raskin P. Diabetic ketoacidosis in type 1 and type 2 diabetes mellitus: clinical and biochemical differences. Arch Intern Med 2004;164:1925-31.

11 Balasubramanyam A, Zern JW, Hyman DJ, et al. New profiles of diabetic ketoacidosis: type 1 vs type 2 diabetes and the effect of ethnicity. Arch Intern Med 1999;159:2317-22.

12 Magee MF, Bhatt BA. Management of decompensated diabetes. diabetic ketoacidosis and hyperglycemic hyperosmolar syndrome. Crit Care Clin 2001:17:75-106.

13 Wang ZH, Kihl-Selstam E, Eriksson JW. Ketoacidosis occurs in both Type 1 and Type 2 diabetes--a population-based study from Northern Sweden. Diabet Med 2008;25:867-70.

14 Rashid MO, Sheikh A, Salam A. Diabetic ketoacidosis characteristics and differences in type 1 versus type 2 diabetes patients, 2017. Available: http://ecommons.aku.edu/ [Accessed 2 Feb 2021]

15 Barski L, Nevzorov R, Jotkowitz A, et al. Comparison of diabetic ketoacidosis in patients with type-1 and type-2 diabetes mellitus. Am J Med Sci 2013;345:326-30.

16 Seth P, Kaur H, Kaur M. Clinical profile of diabetic ketoacidosis: a prospective study in a tertiary care hospital. J Clin Diagn Res 2015;9:OC01-4.

17 Ahmed M, McKenna MJ, Crowley RK. Diabetic ketoacidosis in patients with type 2 diabetes recently commenced on SGLT-2 inhibitors: an ongoing concern. Endocr Pract 2017;23:506-8.

18 Hamblin PS, Wong R, Ekinci El, et al. Sglt2 inhibitors increase the risk of diabetic ketoacidosis developing in the community and during hospital admission. J Clin Endocrinol Metab 2019;104:3077-87.

19 Kempegowda P, Melson E, Johnson A, et al. Effect of COVID-19 on the clinical course of diabetic ketoacidosis (DKA) in people with type 1 and type 2 diabetes. Endocr Connect 2021;10:371-7.

20 Misra S, Barron E, Vamos E, et al. Temporal trends in emergency admissions for diabetic ketoacidosis in people with diabetes in England before and during the COVID-19 pandemic: a populationbased study. Lancet Diabetes Endocrinol 2021;9:671-80.

21 Misra S, Khozoee B, Huang J, et al. Comparison of diabetic ketoacidosis in adults during the SARS-CoV-2 outbreak and over the same time period for the preceding 3 years. Diabetes Care 2021;44:e29-31.

22 Fowler MJ. Microvascular and macrovascular complications of diabetes. Clinical Diabetes 2008;26:77-82.

23 Davis JW, Chung R, Juarez DT. Prevalence of comorbid conditions with aging among patients with diabetes and cardiovascular disease. Hawaii Med J 2011;70:209-13.

24 Zoungas S, Woodward M, Li Q, et al. Impact of age, age at diagnosis and duration of diabetes on the risk of macrovascular and microvascular complications and death in type 2 diabetes. Diabetologia 2014:57:2465-74.

25 Taha Radhi H. Characteristics of diabetic ketoacidosis in adult patients in Bahrain, 2020.

26 Chen Y-L, Weng S-F, Yang C-Y, et al. Long-Term risk of stroke in type 2 diabetes patients with diabetic ketoacidosis: a populationbased, propensity score-matched, longitudinal follow-up study. Diabetes Metab 2017;43:223-8.

27 Signorello LB, Schlundt DG, Cohen SS, et al. Comparing diabetes prevalence between African Americans and whites of similar socioeconomic status. Am J Public Health 2007;97:2260-7.

28 Willi SM, Miller KM, DiMeglio LA, et al. Racial-ethnic disparities in management and outcomes among children with type 1 diabetes. Pediatrics 2015;135:424-34.

29 Spanakis EK, Golden SH. Race/ethnic difference in diabetes and diabetic complications. Curr Diab Rep 2013;13:814-23.

30 Black SA. Diabetes, diversity, and disparity: what do we do with the evidence? Am J Public Health 2002;92:543-8.

31 Lebovitz HE, Banerji MA. Ketosis-Prone diabetes (Flatbush diabetes): an emerging worldwide clinically important entity. Curr Diab Rep 2018:18:120.

32 Kempegowda P, Chandan JS, Coombs B, et al. Regular performance feedback may be key to maintain good quality DKA management: results from a five-year study. BMJ Open Diabetes Res Care 2019;7:695

33 Kempegowda P, Coombs B, Nightingale P, et al. Regular and frequent feedback of specific clinical criteria delivers a sustained improvement in the management of diabetic ketoacidosis. Clin Med 2017;17:389-94. 